\title{
Java-Based Grid Service Spread and Implementation in Remote Sensing Applications
}

\author{
Yanguang Wang ${ }^{1}$, Yong Xue ${ }^{1,2, *}$, Jianqin Wang ${ }^{1}$, Chaolin $\mathrm{Wu}^{1}$, Yincui $\mathrm{Hu}^{1}$, \\ Ying Luo ${ }^{1}$, Shaobo Zhong ${ }^{1}$, Jiakui Tang ${ }^{1}$, and Guoyin Cai ${ }^{1}$ \\ ${ }^{1}$ State Key Laboratory of Remote Sensing Science, Jointly Sponsored by the Institute of \\ Remote Sensing Applications of Chinese Academy of Sciences and Beijing Normal University, \\ Institute of Remote Sensing Applications, Chinese Academy of Sciences, P.O. Box 9718, \\ Beijing 100101, China \\ ${ }^{2}$ Department of Computing, London Metropolitan University, 166-220 Holloway Road, \\ London N7 8DB, UK \\ \{wyg_nju@hotmail.com,y.xue@londonmet.ac.uk\}
}

\begin{abstract}
Remote sensing applications often concern very large volumes of spatio-temporal data, the emerging Grid computing technologies bring an effective solution to this problem. The Open Grid Services Architecture (OGSA) treats Grid as the aggregate of Grid service, which is extension of Web Service. It defines standard mechanisms for creating, naming, and discovering transient Grid service instances; provides location transparency and multiple protocol bindings for service instances; and supports integration with underlying native platform facilities. It is not effective used in data-intensive computing such as remote sensing applications because its foundation, Web Service, is not efficient in scientific computing. How to increase the efficiency of the grid services for a scientific computing? This paper proposes a mechanism Grid service spread (GSS), which dynamically replant a Grid service from a Grid node to the others. We have more computers to provide the same function, so less time can be spent completing a problem than original Grid system. This paper also provides the solution how to adept the service duplicate for the destination node's Grid environment; how each service duplicate communicates with each other; how to manage the lifecycle of services spread etc. The efficiency of this solution through a remote sensing application of NDVI computing is demonstrated. It shows that this method is more efficient for processing huge amount of remotely sensed data.
\end{abstract}

\section{Introduction}

With the development of modern space remote sensing technology, the sensors have got a great increment in spatio-resolution and spectrum-resolution, and have made huge volumes of data for our remote sensing applications. While today's PC is faster than the Cray supercomputer of 10 years ago, it is still often inadequate to provide a

\footnotetext{
${ }^{*}$ Corresponding author.
} 
satisfying computing power, and at the same time the resources of supercomputers are very precious and expensive. Grid computing can give a good solution (Foster and Kesselman 1999). Grid computing technology is being developed to solve two kinds of problems. First, there is much resource wasting in the Internet. Such resources include processing cycles, disk space, data and network. Second, the integration of different systems deployed in a large company tends to be difficult. We need standard technology and platform to support such integration.

To solve this problem, Grid computing considers all the available resource in the network as a "super computer". User can transparently use and manage all these resources. Grid computing also provides a series of standard to integrate heterogeneous systems.

Since 2002, Open Grid Service Architecture (OGSA) which integrates the Globus has stood with the Web Service standard, and is going to be the unified standard for the Grid computing. The basic concept of OGSA is essentially a Web service with improved functionalities and behaviors. Web service is selected because it is a more suitable candidate for Internet scale application compared to other distributed computing technologies, such as CORBA, RMI and EJB (Ceram 2002). First, web service is based on a collection of open standards, such as XML, SOAP, WSDL and UDDI. It is platform independent and programming language independent because it uses standard XML language. Second, web service uses HTTP as the communication protocol. That is a big advantage because most of the Internet's proxies and firewalls will not mess with HTTP traffic. However, Web service is not powerful to build complex applications. It lacks some functionality, such as lifecycle management, notification and persistency. And web service is stateless which means it can't remember what has been done from one invocation to another.

Grid service provides more versatile functionality than web service. We will cover these functionalities in Section 2. Although Grid services get many advantages from Web Services, at the same time they also inherit the low efficiency in scientific computing of Web Service. This paper will propose a mechanism - Grid Services Spread (GSS). It allows the Grid services on a Grid node to extend dynamically to the others in order to increase the number of the nodes serving for the same task. We will focus on this mechanism in details in Section 3. At present, the implementation is based on Java. In Section 4, we will apply this mechanism for a remote sensing application to demonstrate how it works and what it can give us.

\section{Background About Grid Service and Globus Toolkit 3}

A computational Grid is a hardware and software infrastructure that provides dependable, consistent, pervasive, and inexpensive access to high-end computational capabilities. And the latest development of Grid technology gives some progress, that Grid computing is concerned with coordinated resource sharing and problem solving in dynamic, multi-institutional virtual organizations, to the definition.

Comparing Web Services, Grid services has been created with the advatanges including transient service which make the life of the serive can be managed by users and service data which indicates the characters of the service, lifecycly management 
and notification. These adtantages are the tools which we use for building the Grid services spread.

The Globus Toolkit (http://www.globus.org) developed by Argon National Laboratory, University of Chicago and University of Southern California, has become the industrial standard Grid middleware. The famous projects, which used and are using Globus Toolkit, include SF-Express, NASA OVERFLOW-D2, X-ray CMT, Cactus, MM5, National Technology Grid, The European DataGrid, NASA Information Power Grid, ASCI Distributed Resource Management (DRM) Testbed, etc. Globus Toolkit 3 (GT3) is the integrate of the original Globus Toolkit and Web Services, and it implements The Open Grid Services Infrastructure (OGSI) specification, which is the technical specification of Grid services, as an extension to Apache Axis (an implementation of SOAP).

GT3 core implements OGSI, it is a very important part in GT3. GT3 Base Services layer maily include Globus Resource Allocating Mangagement (GRAM in short), Index Service and Reliable File Transfer Service (RFT) which are very important to Globus Toolkit and GSS. GT3 Data Service layer includes Replica Management, which is very useful in applications that have to deal with very big sets of data. When working with large amount of data, we're usually not interested in downloading the whole thing, we just want to work with a small part of all that data. Replica Management keeps track of those subsets of data we will be working with. GRAM handles job submission and management. Index Services are used in discovering services like UDDI in Web Services. RFT allows us to perform large file transfer between the client and the Grid Service. In GT3 core, user does not subscribe to the service instance, but to each service data element. This fine-grained notification may reduce network traffic and improve the system performance.

Grid Service has plenty of contents, but the purpose of this paper is not describing Grid Service in details. More knowleges about Grid Service can be obtained in the The Globus Toolkit 3 Programmer's Tutorial (Sotomayor 2004).

Other Grid Service layer is maintained for the services made by user, for example remote sensing application. These services are built on the Core, Base Service and the Data Service in general. Security is an important factor in Grid-based applications,but this paper doesn't concern the security subject at this stage of study.

\section{Problems and Solution}

The biggest problem of Grid service solving data-intensive computing is that the overhead of remote procedure call is very high. The main reason is that Web Services are based on those standard interoperation technologies,such as XML, SOAP, UDDI which use XML text datastreams.They need plenty of serialization and unserialization. Web Services are necessary to Grid service for the privilege of platform independent, programming language independent and going through Internet proxies and firewalls.This paper will propose a method - Grid Services Spread, which allow a Grid service extend to the other Grid nodes so that more PCs provide their computing resource to the same client request.The request naturally will be completed in shorter time,especially for the application using vast volumes of data. 


\subsection{Design}

The main purpose of GSS is that let more Grid nodes dynamically join to serving for one application. The GSS consists of, in fact, two Grid services source GSS (SGSS) and destination GSS (DGSS). SGSS is in charge of packing all binary code of the service spread, and then sending it to DGSS on the other Grid nodes. DGSS unpacks the bundle from the SGSS and deploys all binary codes to correct paths as the information provided by SGSS. RS Grid Service (Remote Sensing Grid Service) on Node A is called Source Grid Service in GSS, and RS Grid Service Duplicate is called Service Duplicate. Service Duplicate is, in fact, a grid service which have the same functionalities with its Source Grid Service and more characters, such as transience that its lifecycle depends on requests from clients.

More computing resource can been used dynamically with GSS. In Figure 1, Node A only use NDVI services on Node B without GSS, while with GSS Node A can ask SGSS on Node B to copy NDVI Grid service to the other nodes, such as Node C, Node D, and then the NDVI Grid Service Client on Node A can send NDVI Service request to the NDVI Service duplicates on Node $\mathrm{C}$ and $\mathrm{D}$ by the spread information from SGSS on Node B. Three services will be working for the client at a same time, at last the client will integrate the result from each service.

\subsection{Challenges and Solutions}

A Grid service need user to write the following three parts, Service interface which are GWSDL file, Service implementation which are based on Java in this paper, and the Deployment Description (WSDD). With help of them and ANT, which is a java build tool, WSDD and the final java classes are encapsulated to a GAR file (Grid Archive). ANT deploys this GAR file to the Grid services container. So, how to realize GSS? We meet the following challenges.

\section{Which files should be packed by SGSS?}

Before copying a Grid service to the other node, the necessary files about this service should be collected. Which files should be selected? This problem involves two conditions. One is the original GAR file for the service exists. In this case, it is only required to send this GAR file to DGSS as the pack. This situation is very realistic and important. The other is the GAR file does not exist or we do not know where the GAR file is. We find there are some information about the service in undeploy directory. For a simple application, it is possible to make out GAR file because we can find where the class files and the WSDL files are, but for a complex one, it may not be so. The best method is maintaining GAR file in a specific position and using a file to keep the mapping between the GSH of a Grid service and where its GAR file is.

\section{What kind are those services duplicate on the other nodes? Persistent or Transient?}

A Grid service duplicate should be destroyed when the application it serves for is finished, or there are more and more Grid service duplicates running on the node and holding plenty of resources. Therefore those service duplicates should be transient. Who decide the lifecycles of the service duplicates? The service duplicates can be destroyed only when the service client receives satisfactory result. 


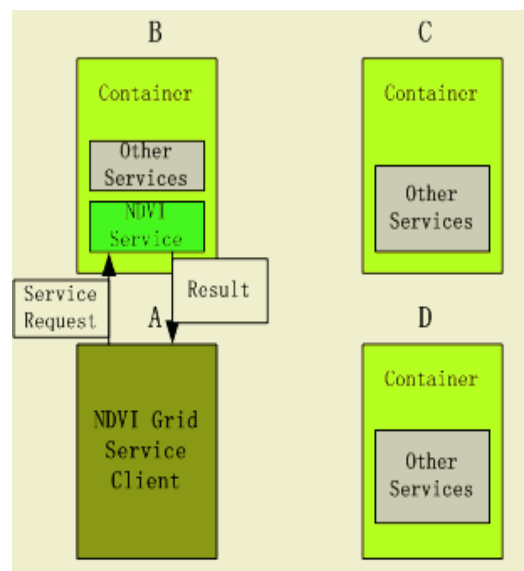

Without GSS

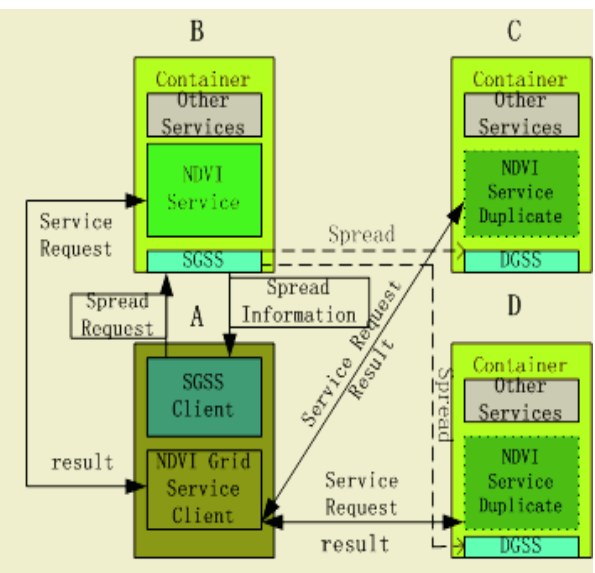

With GSS

Fig. 1. The contrast of NDVI service without and with GSS

How to deploy and undeploy the service duplicates dynamically without restarting the container?

We find that GT3 does not support dynamic service deployment at present through our experiments. A new Grid service deployment is always available only by restarting the container. Our experiments indicate that it's mainly an Axis (a SOAP engine) problem. We can realize dynamic Grid service deployment in GT3 if Axis notices the new services and makes them available to the Grid. Each WAR (Web Archive) contains a fresh copy of the axis/ogsa web-app and the new services are made available when this web-app starts up. We can stop and start this web-app to make our services reinitialize and re-read their configuration files.

\section{Using GSS in an Application of Remote Sensing - NDVI}

Our goal is to test the efficiency of using GSS for Earth surface geophysical parameters determination form remote sensing data. Vegetation indices (VIs) are spectral transformations of two or more bands designed to enhance the contribution of vegetation properties and allow reliable spatial and temporal inter-comparisons of terrestrial photosynthetic activity and canopy structural variations. As a simple transformation of spectral bands, they are computed directly without any bias or assumptions regarding plant physiognomy, land cover class, soil type, or climatic conditions. They allow us to monitor seasonal, inter-annual, and long term variations of vegetation structural, phenological, and biophysical parameters and are important parameter to various kinds of local, regional, and global scale models, including general circulation and biogeochemical models. Numerous studies have shown the utility of the Vegetation Indices (VIs) from climate studies to famine early warning 
detection, epidemiology and renewable natural resources management. The NDVI has also been shown to be highly correlated with net primary production (NPP).

The Normalized Difference Vegetation Index (NDVI) (Tucker 1979, Jackson et al. 1983), which is related to the proportion of photo synthetically absorbed radiation, is calculated from atmospherically corrected reflectance from the visible and near infrared remote sensing sensor channels as:

$$
(\mathrm{CH} 2-\mathrm{CH} 1) /(\mathrm{CH} 2+\mathrm{CH} 1)
$$

Where the reflectance values are the surface bidirectional reflectance factors for MODIS bands $1(620-670 \mathrm{~nm})(\mathrm{CH} 1)$ and $2(841-876 \mathrm{~nm})(\mathrm{CH} 2)$. The NDVI is successful as a vegetation measure in that it is sufficiently stable to permit meaningful comparisons of seasonal and inter-annual changes in vegetation growth and activity.

\subsection{Experiment}

In this experiment, we used 1000 MODIS images (1000x1200 pixels), and each image is about $1.1 \mathrm{M}$. We calculate the NDVI as batches using these MODIS images through different approaches: single PC without NDVI grid service, NDVI grid service on only one node and NDVI grid service spreading to 5 PCs with GSS.

Our test bed consists of 1 PC running Linux9.0 and 5 PCs running Windows 2000 operating system. It makes a good advantage of the individual PCs, especially in a work group, to constitute a Grid system based on GT3 core and GT3 Gars, which include RFT, MMJFS, GRAM and MDS etc. Table 1 gives the configuration information of our test bed on GT 3.

NDVI Service Client program on Tgp2.tgp requests the SGSS Service on wang.tgp to spread the NDVI Service. Then the SGSS returns GSHs of the NDVI Service duplicates that has been spread to Cai.tgp, Ly.tgp, Zhong.tgp and Hu.tgp. When getting the information, Client program divides remotely sensed data (MODIS data in the experiment) in geometric parallelism and send request to all the NDVI Services including original one and duplicates.

\subsection{Result and Analysis}

This experiment involved three cases:

1) We calculate NDVI with single PC without Globus Toolkit, using varying amount of data from $11 \mathrm{MB}$ to $1100 \mathrm{MB}$ (form 10 images to 1000 images).

2) We do that with Grid Service, but without Grid Service Spread mechanism. NDVI Grid Service only runs on one PC. The amount of data still is varied from 11MB to $1100 \mathrm{MB}$.

3) We use Grid Service Spread. The situation of utilizing data still is so.

This experiment was done for 10 times successively and we give the average value in Table 2, which is the statistics of the results of the three cases. We define the efficiency of GSS is the ratio of these two execution times: 
Table 1. The configurations of GT 3 test bed

\begin{tabular}{|c|c|c|c|c|c|}
\hline $\begin{array}{l}\text { Host } \\
\text { name }\end{array}$ & IP address & $\mathrm{CPU}$ & OS & $\begin{array}{l}\text { Globus } \\
\text { Toolkit }\end{array}$ & Description \\
\hline Tgp2.tgp & 192.168 .0 .200 & $1.7 \mathrm{GHz}$ & $\begin{array}{l}\text { Win } \\
2000\end{array}$ & $\begin{array}{l}\text { GT } 3 \\
\text { core and } \\
\text { Gars }\end{array}$ & Service Client \\
\hline Wang.tgp & $\begin{array}{l}192.168 .0 .1 \\
159.226 .117 .121\end{array}$ & $2.0 \mathrm{GHz}$ & Linux9.0 & $\begin{array}{l}\text { GT } 3 \\
\text { core and } \\
\text { Gars }\end{array}$ & $\begin{array}{l}\text { NDVI Service } \\
\text { SGSS }\end{array}$ \\
\hline Hu.tgp & 192.168.0.119 & $2.6 \mathrm{GHz}$ & Win 2000 & $\begin{array}{l}\text { GT3 } \\
\text { core and } \\
\text { Gars }\end{array}$ & $\begin{array}{l}\text { Service } \\
\text { Duplicate } \\
\text { DGSS }\end{array}$ \\
\hline Ly.tgp & 192.168.0.120 & $2.0 \mathrm{GHz}$ & Win 2000 & $\begin{array}{l}\text { GT3 } \\
\text { core and } \\
\text { Gars }\end{array}$ & $\begin{array}{l}\text { Service } \\
\text { Duplicate } \\
\text { DGSS }\end{array}$ \\
\hline Cai.tgp & 192.168.0.110 & $2.6 \mathrm{GHz}$ & $\begin{array}{l}\text { Win } \\
2000\end{array}$ & $\begin{array}{l}\text { GT } 3 \\
\text { core and } \\
\text { Gars }\end{array}$ & $\begin{array}{l}\text { Service } \\
\text { Duplicate } \\
\text { DGSS }\end{array}$ \\
\hline Zhong.tgp & 192.168.0.19 & $2.6 \mathrm{GHz}$ & $\begin{array}{l}\text { Win } \\
2000\end{array}$ & $\begin{array}{l}\text { GT } 3 \\
\text { core and } \\
\text { Gars }\end{array}$ & $\begin{array}{l}\text { Service } \\
\text { Duplicate } \\
\text { DGSS }\end{array}$ \\
\hline
\end{tabular}

Table 2. The results of the three cases

\begin{tabular}{|c|c|c|c|c|}
\hline Cases Amount of Data & $\begin{array}{l}\text { 10 Images } \\
(11 \mathrm{MB})\end{array}$ & $\begin{array}{l}100 \\
\text { Images } \\
(110 \mathrm{MB})\end{array}$ & $\begin{array}{l}500 \\
\text { Images } \\
(550 \mathrm{MB})\end{array}$ & $\begin{array}{l}\text { 1000 Images } \\
(1100 \mathrm{MB})\end{array}$ \\
\hline Without Grid Service (1 PC) & $3.5 \mathrm{~s}$ & $42.9 \mathrm{~s}$ & $321.8 \mathrm{~s}$ & $1093.8 \mathrm{~s}$ \\
\hline $\begin{array}{l}1 \text { PC providing Grid Service } \\
\text { without GSS }\end{array}$ & $8.4 \mathrm{~s}$ & $49.2 \mathrm{~s}$ & $342.4 \mathrm{~s}$ & $1128.4 \mathrm{~s}$ \\
\hline $\begin{array}{l}\text { NDVI Grid Service spreading } \\
\text { to } 5 \text { PCs with GSS }\end{array}$ & $15.6 \mathrm{~s}$ & $24.8 \mathrm{~s}$ & $84.3 \mathrm{~s}$ & $253.7 \mathrm{~s}$ \\
\hline
\end{tabular}

Case 2 is little slower than case 1 because of the serialization and unserialization of Grid service, but the difference varies little with the increase of the amount of data. In case 3, GSS even use more time to calculate NDVI when the amount is $11 \mathrm{M}$ for it is also a Grid service in fact. And the advantage of GSS is not huge when 100 images are used, but it is very surprising effective when it comes to $550 \mathrm{M}$ (efficiency = 3.7) and $1100 \mathrm{M}$ (efficiency $=4.3$ ). We are convinced that we will have a better efficiency if the service can be spread to a bigger range. For a specific range of GSS, the efficiency will be better if the amount of data processed is larger. 


\section{Conclusion}

Grid Service, the combination of Grid Technologies and Web Service, is a trend because it can really change the whole Internet into a huge real Grid system. But its performance is not satisfying in scientific computing, especially in data-intensive. Grid Service Spread mechanism can help us use more computing resource to service for our applications dynamically. With the help of GSS, we will have more powerful computing abilities and Throughput. It shows that this method is more efficient for processing huge amount of remotely sensed data.

\section{Acknowledgements}

This publication is an output from the research projects "CAS Hundred Talents Program" and "Monitoring of Beijing Olympic Environment" (2002BA904B07-2) and "Remote Sensing Information Processing and Service Node" funded by the MOST, China and "Aerosol fast monitoring modeling using MODIS data and middlewares development" (40471091) funded by NSFC, China.

\section{References}

Ceram, E., 2002, Web Services Essentials, (Sebastopol: O’Reilly \& Associates, Inc), pp, 7-24.

Foster, I., Kesselman, C. 1999, The Grid: Blueprint for a New Computing Infrastructure (San Francisco: Morgan Kaufmann), pp, 16-50

Foster, I., Kesselman, C. and Tuecke, S., 2001, The Anatomy of the Grid: Enable Scalable Virtual Organizations. Available online at http://www.globus.org/reserch/ papers.html (accessed 10 Month 2004)

Foster, I., Kesselman, C., Nick, J., Tuecke, S., 2002, The Physiology of the Grid. Available online at http://www.Gridforum.org/ogsi-wg/drafts/ogsa_draft2.9_2002-06-22.pdf (accessed 10 Month 2004)

Jackson, R.D., P.N. Slater, and P.J. Pinter, 1983, Discrimination of growth and water stress in wheat by various vegetation indices through clear and turbid atmospheres. Remote Sensing of the Environment, 15, 187-208.

Sotomayor, B., 2004, The Globus Toolkit 3 Programmer's Tutorial, Available online at http://www.casa-sot $\backslash$ omayor.net/gt3-tutorial (accessed 10Month 2004)

Tucker, C. J., 1979, Red and photographic infrared linear combinations for monitoring vegetation. Remote Sensing of the Environment, 8, pp, 127-150.

Tuecke, S., Czajkowski, K., Foster, I., Frey, J., Graham, S., and C. Kesselman, C., 2002, Grid Service Specification. Available online at http://www.Gridforum.org/ogsi-wg/drafts/ GS_Spec_draft03_2002\-07-17.pdf(accessed 10 Month 2004) 